DOI: $10.17725 /$ rensit.2019.11.321

\title{
Binding of short DNA oligonucleotides to the surface of reduced graphene oxide to create biological sensors
}

Ivan A. Komarov, Alexander N. Kalinnikov, Sergey N. Shcherbin, Vyacheslav A. Seleznev

Bauman Moscow State Technical University, http://www.bmstu.ru/

Moscow 105005, Russian Federation.

Olga M. Antipova

Lomonosov Moscow State University, https:/ /www.msu.ru/

Moscow, 119991 Russian Federation

Nikolay S. Struchkov

National Research University of Electronic Technology, https://miet.ru/

124498, Moscow, Zelenograd, Russian Federation.

E-mail:ikomarov@emtc.ru,alexandr.kalinnikov@emtcru,sshcherbin@emtc.ru,vseleznev@emtc.ru,antipovachem@gmail. com,struchkov.nikolaj@gmail.com

Received 31.10.2019, peer reviewed 11.11.2019, accepted 18.11.2019

Abstract. The study examined the possibility of immobilization of short DNA oligonucleotides (aptmers) on the surface of reduced graphene oxide, with the aim of forming a bio-sensitive sensory layer. The features of laser reduction of graphene oxide were considered, and the parameters of reduction were determined, which were implicated in achieving partial reduction. The possibility of immobilization of aptamers on graphene oxide with a low degree of reduction and the response of sensory structures formed on the basis of this basis is shown. A significant difference between the response of the sensors to the effects of thrombin (target protein) and albumin (comparison protein) was demonstrated.

Keywords: aptamers, biosensors, immobilization, nucleotides, graphene oxide

UDC 620.3

Acknowledgements: This work was financially supported by the grant of the Ministry of Education and Science of the Russian Federation No. 14.574.21.0182 (unique identifier RFMEFI57417X0182).

For citation: Ivan A. Komarov, Olga M. Antipova, Nikolay S. Struchkov, Alexander N. Kalinnikov, Sergey N.

Shcherbin, Vyacheslav A. Seleznev. Binding of short DNA oligonucleotides to the surface of reduced graphene oxide to create biological sensors. RENSIT, 2019, 11(3):321-330; DOI: 10.17725/rensit.2019.11.321.

\section{Contents}

1. INTRODUCTION (321)

2. Materials AND MEthods (323)

3. Results AND discussion (324)

3.1. AFM IMAGING OF GRAPHENE OXIDE FILMS (324)

3.2. INVESTIGATION OF THE FEATURES OF THE RECOVERY OF GRAPHENE OXIDE FILM (325)

3.3. IMMOBILIZATION OF APTAMERS (325)

3.4. STUDY OF THE FEATURES OF IMMOBILIZATION (326)

3.5. SENSOR RESPONSE RESEARCH (328)

4. Conclusion (328)

REFERENCES (329)

\section{INTRODUCTION}

The market for electronic devices is growing rapidly and shifting more and more towards the personalized devices with large number of different sensors. Among them devices for health monitoring are most claimed. In addition, 5th generation mobile networks that allow data collection and transfer from a large number of different sensors have recently appeared. Another important trend of the world healthcare is remote patient control without the need to visit a hospital. Based on the abovementioned trends, we can conclude that in the nearest future diagnostics 
of some diseases will be held completely online. And in the far future diagnostics of the wide spectrum of diseases will be made remotely that requires sophisticated systems for personal diagnostic. One of the main requirements for such systems is the creation of sensors that can directly measure presence of specific pathogens since far from all diseases and pathologies can be determined by such indirect measurements as heart rate, pressure or ECG (today the most sophisticated smart bracelets and watches allow such measurements). Preferably new generation of biological sensors should detect the presence of pathogens or their markers in physiological fluids or human breathing. The development of affordable sensors of this kind could greatly simplify the chain that include taking medical tests; movement samples from the clinic to the laboratory, processing samples in the laboratory by qualified personnel, results transfer to the patient, results transfer to the doctor. According to last advances in data transfer technologies and in case of the presence of highly sensitive and selective sensors the abovementioned chain can be reduced to sample deposition on sensors and transfer results of measurement to the patient and doctor. Great advance of such approach is that results can be obtained in a few minutes.

Based on foregoing, it is possible to determine the basic requirements for biosensors: high accuracy, selectivity and velocity of the test with minimal usage skills, possibility of on-site detection and low cost of the tests.

Taking into account the above requirements for the biosensor, one of the most promising materials is reduced graphene oxide, obtained by reduction of suspensions, films, or graphene oxide powders. Due to the presence of functional groups and relatively high conductivity, reduced graphene oxide can be successfully used both for the formation of a sensitive layer and transducer [1]. The advantage of graphene oxide is its good dispersibility, which makes possible graphene oxide deposition on various substrates by liquid methods, in particular, drop-casting [2], spin-coating [3] or aerosol deposition [4]. The reduction of graphene oxide can be carried out with the retention of a part of the functional groups, which subsequently can be used to immobilize bioavailable agents [5].

Several mechanisms of reduction of graphene oxide can be distinguished: thermal [6], chemical [7] and photochemical realized using UV radiation [8], as well as methods based on the use of lasers [9].

In this paper we showed the possibility of graphene oxide films formation with further local reduction of these films using laser irradiation. The possibility of controlled reduction of graphene oxidewith the presence of a certain number of functional groups and conductivity is also shown. Moreover, we immobilized short DNA oliguncleotides (aptamers) to reduced graphene oxide film through a modified EDC/HNS reaction. Aptamers as biosensitive agents have such advantages over antibodies as higher stability, much simpler and cheaper synthesis [10-14]. As a final point we demonstrated selective response of sensors structures based on reduced graphene oxide with the binded aptamers to the target and reference proteins. 


\section{MATERIALS AND METHODS}

As the substrate for graphene oxide film deposition we used polyethylene terephthalate (PET) with a thickness of $175 \mu \mathrm{m}$ and a lateral size of the substrate of $40 \times 40 \mathrm{~mm}$. We used PET is due to the relatively high melting point, significantly exceeding $250^{\circ} \mathrm{C}$ that makes possible the heat treatment of the deposited films, and acceptable mechanical characteristics, at a low cost. The preparation of the substrates for the deposition of graphene oxide consisted in mechanical cleaning in 2-propanol (ECOS-1, Extra Pure, TU 2631-064-44493179-01).

An aqueous suspension of graphene oxide $(4.7 \mathrm{mg} / \mathrm{ml})$ obtained by the advanced Hammers method (MIP Graphene LLC, Russian Federation) [15] was diluted with deionized water to a concentration of 1.5 $\mathrm{mg} / \mathrm{ml}$ and deposited on PET surface by spin-coating with the number of iterations from 5 to 15 . Obtained films were dried at a temperature of $110^{\circ} \mathrm{C}$ for 20 minutes. The thickness of the obtained films was in range from 40 to $100 \mathrm{~nm}$ (according to atomic force microscopy).

Local restoration of the graphene oxide film was carried out by an automated setup which included a diode laser (445 nm wavelength) and a motorized sample table [16]. Control of the laser power in the range of $10-400 \mathrm{~mW}$ was carried out by pulsewidth modulation with a frequency of $30 \mathrm{MHz}$. The pulse width ranged from 1 to $20 \mu \mathrm{s}$. The processing time at the point was $30 \mathrm{~ms}$ (the size of the laser beam was about $\left.1.5 \cdot 10^{3} \mu \mathrm{m}^{2}\right)$.

For the formation of a biosensitive layer, amine modified thrombin aptamers AmTBA with a nucleotide sequence (5'-GGTTGGTGTGGTTGG-3 ') were used. Aptamers were synthesized using standard chemistry of phosphoramidites and purified by liquid chromatography (Apto-Farm, LLC, Russia). The amino group was introduced as a modified thymine residue at the 5 '-end of the DNA sequence. The binding of aptamers to carboxyl groups contained in reduced graphene oxide was carried out using a commercially available electrophilic activator EDC (1-ethyl-3(3-dimethylaminopropyl) -carbodiimide (Sigma-Aldrich, USA) .The reaction was carried out in $100 \mathrm{mM}$ buffer a solution of MES (2- (N-morpholino) ethanesulfonic acid, Sigma-Aldrich, USA), $\mathrm{pH}=6.0$, with the addition of $25 \%$ ethanol, at room temperature in an inert gas atmosphere. The final concentration of the aptamer in the conjugation reaction amounted to 50 $\mu \mathrm{m}$. Binding occurred within 1 hour, after The solution was collected, centrifuged and analyzed by HPLC.

HPLC measurements were performed on an Agilent Technologies 1200 instrument with a DNA PAC 100 chromatographic column (particle diameter $4 \times 250 \mathrm{~mm}$ and 13 $\mu \mathrm{m}$, Thermo Scientific, USA). The mobile phases were as follows: A: $10 \mathrm{mM}$ Tris, $1 \mathrm{mM}$ EDTA, $\mathrm{pH}=7.5,5 \%$ acetonitrile; $\mathrm{B}: 10 \mathrm{mM}$ Tris, $1 \mathrm{mM}$ EDTA, $\mathrm{pH}=7.5,1 \mathrm{M} \mathrm{NaCl}, 5 \%$ acetonitrile. The gradient program included two stages: $0-1 \mathrm{~min}-30 \% \mathrm{~B}, 1-20 \min 30-$ $50 \% \mathrm{~B}$. The column temperature was $60^{\circ} \mathrm{C}$, the flow rate was $1 \mathrm{ml} / \mathrm{min}$. Detection was carried out by absorption at a wavelength of $254 \mathrm{~nm}$.

Raman spectra were obtained on a Centaur U HR combined AFM/Raman complex (NanoScanTechnology Ltd, Russian Federation). 
Fourier-IR spectroscopy was carried out on a Nicolet iS10 FTIR spectrometer (Thermo Fisher Scientific, USA) by the method of impaired total internal reflection.

The surface topography of the graphene oxide films was obtained using a Solver P47Pro scanning probe microscope (NT-MDT, Russia).

Thrombin was used as the target protein, and albumin was used as the reference protein. The response of biosensors to the effects of proteins was recorded using an IPS-16 sensor parameters meter (Pracktik NC Ltd., Russian Federation).

\section{RESULTS AND DISCUSSION}

\subsection{AFM IMAGING OF GRAPHENE OXIDE FILMS}

We measured thickness of the obtained films by atomic force microscopy and made correlation with made correlation with the transmittance of the same films made by spectrophotometry. By the correlation between the spectrophotometric data, it is subsequently possible to make a fast control the thickness of the deposited films. The dependence of film thickness and optical transmittance on the number of deposition iterations in on Fig. 1.

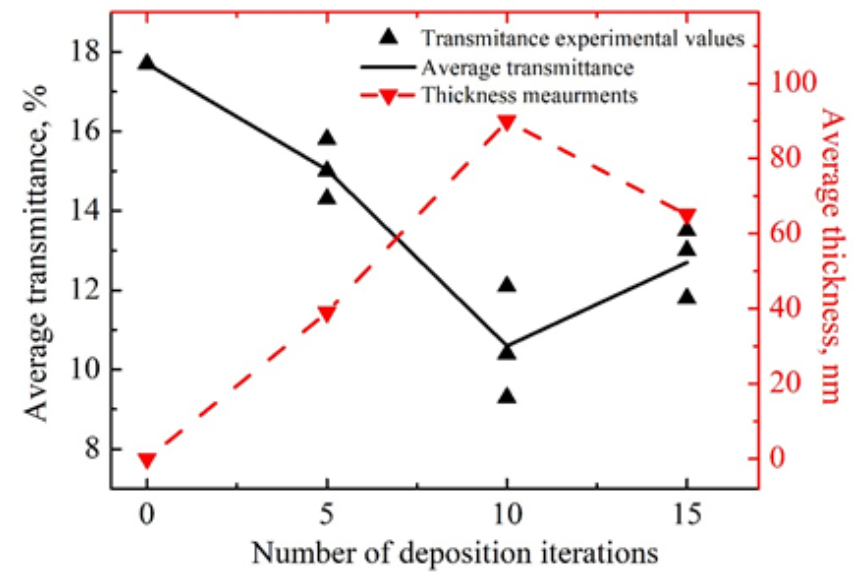

Fig. 1. Dependence of the thickness of the formed films and the optical transmittance on the number of deposition iterations.
The thickness of the obtained films was determined using atomic force microscopy according to the following procedure: a graphene oxide film was partially removed from the substrate with the end face of another PET substrate so that a step formed between the substrate and the graphene oxide film. The height of the step was determined by the surface topography (Fig. 2). The morphology of the film surface is typical for graphene oxide films and is similar to the morphology of the film presented in [17].

Based on the data obtained, it is possible to note the presence of a threshold number of depositions - 10, at which the maximum film thickness is achieved. It is assumed that this threshold is due to the fact that upon depositions the film does not have time to completely dry. In this case the adhesion of the upper layers of the film is less than the centrifugal force for a given solution concentration and substrate rotation speed.

From the obtained data, it can be noted that the film thickness and optical transmittance have a linear dependence on the number of deposition iterations and are inversely proportional to each other. The maximum film thickness is achieved at 10 iterations of deposition and is $90 \mathrm{~nm}$. In this case, the optical transmittance is about $10 \pm 2 \%$, which was used in the further preparation of the sensor structures.

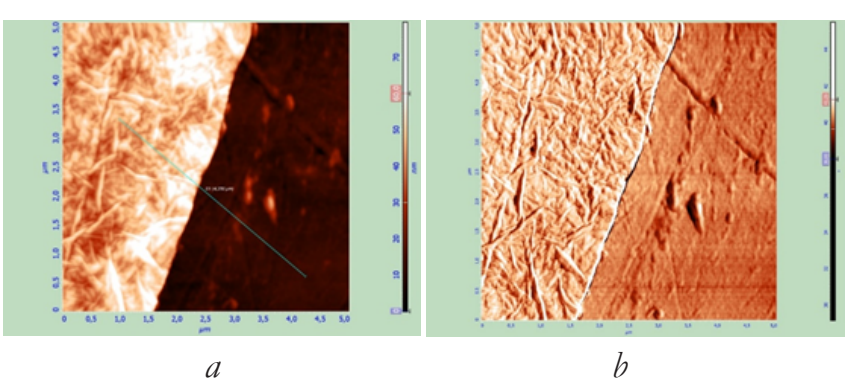

Fig. 2. The topography of the graphene oxide film (a) and the image of the boundary of the graphene oxide film and the PET substrate in the phase contrast mode. 


\subsection{INVESTIGATION OF THE FEATURES OF} THE RECOVERY OF GRAPHENE OXIDE FILMS

The thickest graphene oxide films were used to study the features of reduction by laser irradiation. The films were treated with laser fluence from 15.2 to $59.4 \mathrm{~J} / \mathrm{cm}^{2}$. We assumed that at low fluence it will be weak reduction of graphene oxide but with conductive channels formation and residual amounts of functional groups, that will be sufficient for aptamer binding. From the obtained results (Fig. 3) we can note that the ratio $I_{D} / I_{G}$ is reduced to about 0.4 with increasing fluence to about $37 \mathrm{~J} / \mathrm{m}^{2}$, which means that there is a removal of structural defects, primarily due to removal oxygen-containing functional groups. When fluence is more than $60 \mathrm{~J} / \mathrm{cm}^{2}$ we observed destruction of the film with partial ablation of substrate. The maximum degree of graphitization and according to Raman spectroscopy is achieved at a fluence of $48.3 \mathrm{~J} / \mathrm{cm}^{2}$, which follows from the ratios $I_{D} / I_{G}$ and $I_{2 D} / I_{G}$. But considering the fact that the resistance and ratio $I_{D} / I_{G}$ and $I_{2 D} / I_{G}$ vary in the range from 35 to $50 \mathrm{~J} / \mathrm{cm}^{2}$, it is assumed that the resulting energy is sufficient to heat the graphene oxide film over the

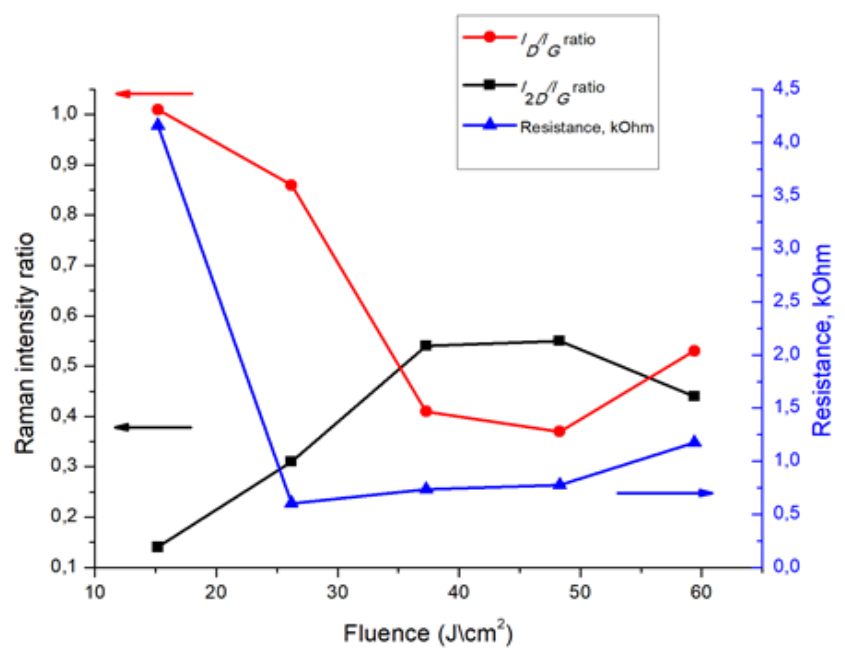

Fig. 3. Dependence of the intensity ratios $D, G$, and $2 D$ of Raman peaks and the resistance of the reduced regions on the laser fuence. entire thickness. Increasing $I_{2 D} / I_{G}$ ratio tells us about structuring of reduced graphene oxide due to the presence turbostratic graphite consisting of several graphene flakes which have a random orientation in the layer. It is interesting to note that there is a significant correlation between the resistance of the reduced areas and the $I_{D} /$ $I_{G}$ ratio, that assumes quasi-linear nature of the energy distribution in the irradiated graphene oxide film.

Based on the data of Raman spectroscopy it can be concluded that the maximum number of functional groups (i.e., structural defects) observed in $15-25 \mathrm{~J} / \mathrm{cm}^{2}$ fluence range but nevertheless reduced graphene oxide film in this case shows conductivity. This fluence values were used in further work for sensor structures formation.

\subsection{IMMOBILIZATION OF APTAMERS}

To study the specific features of the binding of DNA nucleotides to reduced graphene oxide, we used a well-studied aptamer with affinity for thrombin [18] with amino modification (Am-TBA). The nucleotide sequence of this aptamer: 5'-GGTTGGTGTGGT'TGG-3'. As it was shown in [19], TBA recognizes thrombin with very high affinity using its TT loops, and the core structure of the G quadruplex stabilizes the exact location of the loops. In this case we used the 5'-amino-thymidine modification of the aptamer (Am-TBA), due to the fact that the addition of nucleotide residues and functional groups at the 5'-end of the TBA does not change the structure of the aptamer that binds thrombin and does not affect binding to the target protein [20]. The amine group in Am-TBA is necessary for covalent bonding by carboxyl groups 
of the reduced graphene oxide film preactivated with carbodiimide (EDC).

The EDC conjugation method is an extremely common practice for bioconjugation: the coupling reaction proceeds with a quantitative yield. However, when we applied this method to conjugate an amino-modified aptamer derivative with a reduced graphene oxide film, we saw that the hydrophobic surface effect of the reduced graphene oxide plays an important role. We did not get a satisfactory yield with the standard reaction method. We have shown that the addition of $25 \%$ vol. of ethanol to the reaction mixture improves the yield of the reaction.

HPLC analysis of AmTBA amount allows only reactive carboxyl groups to be calculated. However, it is the number of active carboxyl groups that determines the number of conjugated sensitive molecules on the surface of the reduced graphene oxide and, therefore, it determines the effectiveness of the biosensor.

Based on the amount of the aminomodified AmTBA aptamer, we calculated that the specific number of carboxyl groups reaches 29 pmol per $1 \mathrm{~mm}^{2}$, maximum at fluence $42 \mathrm{~J} / \mathrm{cm}^{2}, 32 \mathrm{pmol}$ per $1 \mathrm{~mm}^{2}$, at fluence $35 \mathrm{~J} / \mathrm{cm}^{2}$ and 43 pmol per $1 \mathrm{~mm}^{2}$ films at a minimum fluence of $25 \mathrm{~J} / \mathrm{cm}^{2}$ (Table).

Table

Aptamer conjugation results.

\begin{tabular}{|c|c|c|c|}
\hline $\begin{array}{c}\text { Laser } \\
\text { fluence, } \\
\mathrm{J} / \mathrm{cm}^{2}\end{array}$ & $\begin{array}{c}\text { The } \\
\text { percentage } \\
\text { change in the } \\
\text { concentration } \\
\text { of AmTBA,\% }\end{array}$ & $\begin{array}{c}\text { Quantitative } \\
\text { change in the } \\
\text { quantity of } \\
\text { AmTBA, nmol }\end{array}$ & $\begin{array}{c}\text { The specific } \\
\text { amount of } \\
\text { reactive carboxyl } \\
\text { groups per unit } \\
\text { area, } 10^{12} / \mathrm{nm}^{2}\end{array}$ \\
\hline 25 & 32 & 3.2 & 43 \\
\hline 35 & 29 & 2.9 & 32 \\
\hline 42 & 26 & 2.6 & 29 \\
\hline
\end{tabular}

Thus, we observed a tendency to increase the presence of carboxyl functional groups with a decrease in the laser fluence and determined that laser treatment with a fluence of about $25 \mathrm{~J} / \mathrm{cm}^{2}$ is suitable for aptamer communication and, therefore, for the entire sensor.

\subsection{STUDY OF THE FEATURES OF IMMOBILIZATION}

The Raman spectra from the sensor structures before and after the aptamer interaction are shown in Fig. 4. In these spectra, we can observe $D\left(\sim 1350 \mathrm{~cm}^{-1}\right), G$ $\left(\sim 1600 \mathrm{~cm}^{-1}\right)$ and $2 D\left(\sim 2700 \mathrm{~cm}^{-1}\right)$ bands respectively. It is known that the $D$ band arises due to $A_{1 g}$ symmetry and originates from the zone boundary phonons. The $D$ band is usually attributed to various defects in the graphite lattice. The $G$ band on the other hand is first-order scattering, related to $E_{2 g}$ symmetry and corresponds to the Brillouin zone of crystalline $s p^{2}$ lattices in graphite [21].

Higher ID/IG ratio in the reduced graphene oxide indicates larger defect density $[22,23]$ i.e. including functional groups. Thus in our case we observe large defect presence in the sensitive area of biosensor including functional groups It

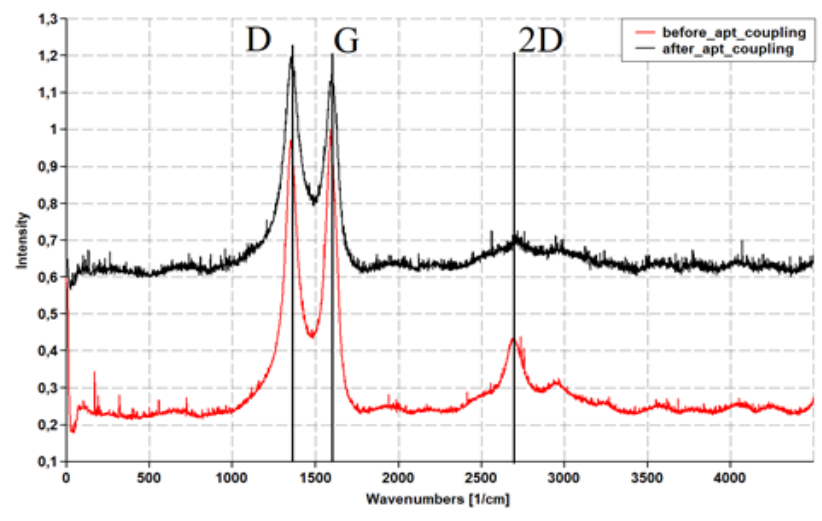

Fig. 4. Raman spectra on the reduced surface of graphene oxide before and after aptamer interaction. 
has been reported that the $2 \mathrm{D}$ peak profile is sharp for pristine graphene, whereas low and wide $2 \mathrm{D}$ peak intensity as compared to $D$ and $G$ peaks indicates higher disorder rate in the GO structure [24]. From figure 5 and abovementioned information on the nature of Raman bands we can make next observations and deduction. One can see that there are 2 main features in spectra after coupling: change in ID/IG ratio (0.98-0.99 before to 0.86-1.13 after aptamer coupling) and decrease of $2 D$ peak intensity. As we assume these effects could be corresponded to increase of defect number due to presence of aptamer-rGO conjugates with the trend and values ID/ IG ratio before and after aptamer coupling very similar to the effects obtained in $[25,26]$. Authors of [26] correspond this effect to the coupling of polyethylenimine (PEI) to the graphene oxide. In our case, such comparison is legal due to low reduction rate of graphene oxide that thus contains different functional groups. Based on abovementioned information we have indirect confirmation of aptamer coupling that is confirmed further by FTIR spectroscopy (Fig. 5).

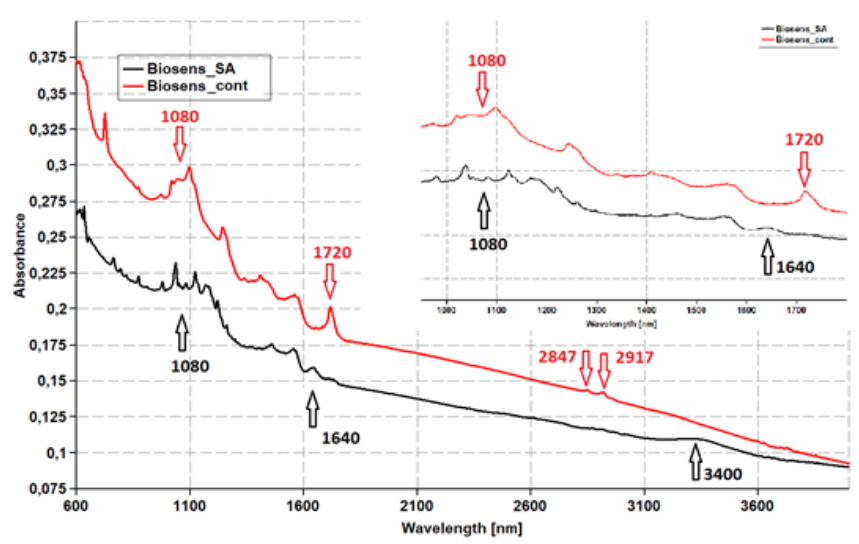

Fig. 5. FTIR spectra of the biosensor in the region of the reduced oxide with and without aptamers applied.
From Fig. 5 we can mention presence of several characteristic peaks we attribute to hydroxyl, carboxyl, carbonyl and epoxide. Broad peak at $\sim 3400 \mathrm{~cm}^{-1}$ could be attributed to $\mathrm{O}-\mathrm{H}$ stretching vibrations, i.e. hydroxyl groups presence [27]. One should note that this peak is clearly observed only in sensitive area after coupling reaction. Minor peaks at $2847 \mathrm{~cm}^{-1}$ (symmetric) and $2917 \mathrm{~cm}^{-1}$ (asymmetric) are $-\mathrm{CH}_{2}$ absorption band that was split into two sub-bands and can be assigned to alkyl moieties (as a result of the reduction of $\mathrm{COOH}$ to $-\mathrm{CH}_{3}$ segment) [28]. Relatively intensive peak at $\sim 1720 \mathrm{~cm}^{-1}$ in case of reduced graphene oxide in contact area should be attributed to carboxyl groups presence, which means that reduction with low fluence doesn't remove all functional groups. On the other hand, large decrease in this peak intensity after aptamer coupling means that we observe reaction between aptamer and carboxyl group. The same situation is described in [29] for 6 arm polyethylene glycol amines conjugated with graphene oxide. According to [30] the peak at $\sim 1720 \mathrm{~cm}^{-1}$ should be attributed to $\mathrm{C}=\mathrm{O}$ carboxyl and carbonyl groups, peak at $1640 \mathrm{~cm}^{-1}$ can be attributed to the skeletal vibrations of un-oxidized graphitic domains. Wide band at about $\sim 1080 \mathrm{~cm}^{-1}$ appears due to hydroxyls [31].

From FTIR spectra we can make a conclusion that after the graphene oxide reduction. A number of functional groups still remain in the film. Moreover, after aptamer coupling, we observe decrease of the carboxyl peak intensity, which means transformation of this groups after coupling reaction. It is that we want and predict for low reduction ratio. 

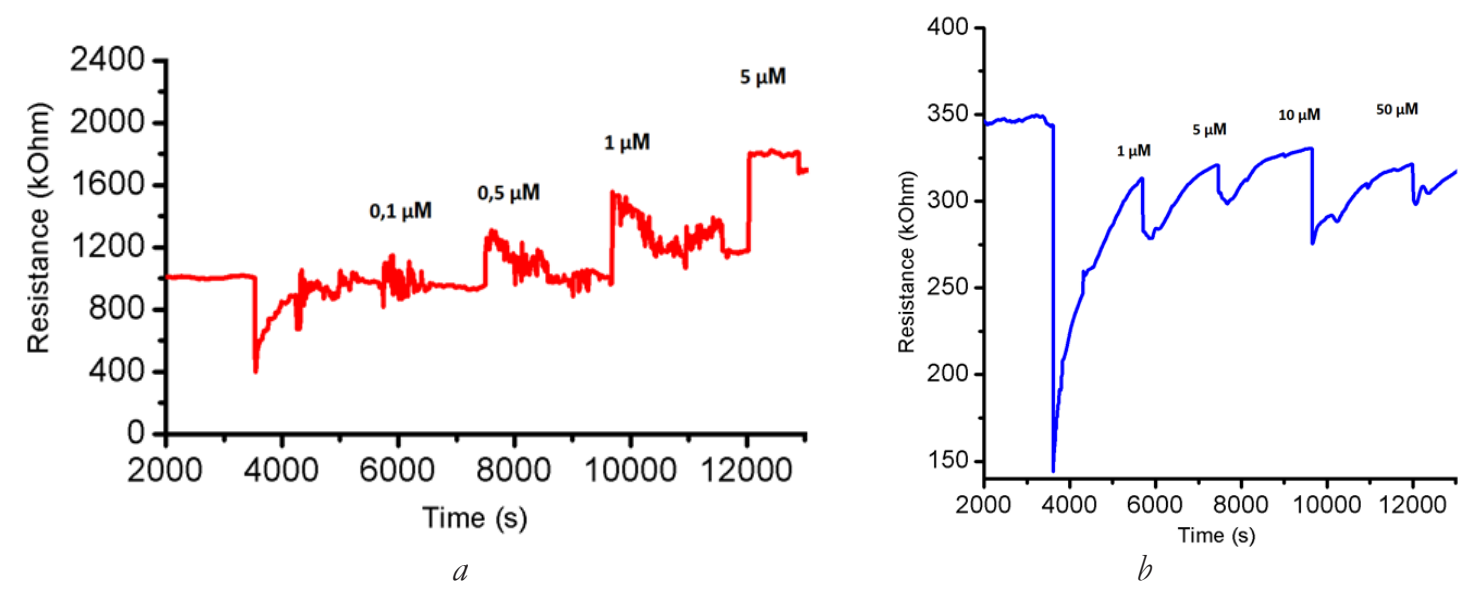

Fig. 6. Response of sensory structures to thrombin (a) and albumin (b).

\subsection{SENSOR RESPONSE RESEARCH}

We exposed the sensitive area with thrombin (target) and albumin proteins (reference) to get a response from the sensor. Sensors with a coupled thrombin-binding aptamer were exposed to thrombin (Fig. 6a) and albumin (Fig. 6b). We observed a significant difference between sensory signals acting on different proteins. The sensors also showed various signs of resistance change,

Fig. 6 shows that there is a reaction to thrombin in which the response increases with increasing concentration of thrombin, while in the case of albumin there is no noticeable difference in response even at concentrations differing by 2 orders of magnitude. The latter fact suggests that albumin interacts nonspecifically with aptamers, whereas in the case of thrombin, it is precisely the selective interaction of the aptamer with thrombin that is observed. In addition, it is noticeable in Fig. $6 a$ that, after reaching the maximum response, a resistance fluctuation is observed with a trend towards a return to the initial resistance. We attribute the fact of resistance fluctuation to protein desorption process, while in case of albumin we observe nonspecific interaction of albumin with aptamers, cause in case of interaction with reduced graphene oxide surface we should observe must observe non-recovery response due strong coupling of $\mathrm{rGO}$ with albumin like in [32]. The detection limit of the developed sensor on thrombin is in range from 1 to $10 \mu \mathrm{M}$.

\section{CONCLUSION}

In this work, we showed the possibility of the formation and controlled reduction of graphene oxide films using laser radiation. The possibility of binding short DNA oligonucleotides (aptamers) with reduced graphene oxide through the carboxyl groups of the latter was shown. The optimal range of fluence values for the reduction of graphene oxide is $15-25 \mathrm{~J} / \mathrm{cm}^{2}$. Optimal binding of aptamers is achieved with minimal fluence. The response of the sensors to the target and reference protein is shown, and there are noticeable differences in the response to thrombin and albumin with a minimum detectable concentration of the first $1 \mu \mathrm{M}$.

\section{ACKNOwLEDGEMENTS:}

This work was financially supported by the grant of the Ministry of Education and Science of the Russian Federation No. 14.574.21.0182 (unique identifier RFMEFI57417X0182). 


\section{REFERENCES}

1. Celik N, Balachandran W, Manivannan N. Graphene-based biosensors: methods, analysis and future perspectives. IET Circuits Devices Syst., 2015, 9:434-445.

2. Xiaoning Tang, Xiansheng Zhang, Huiping Zhang, Xingmin Zhuang and Xiong Yan. Facile dip-coating process towards multifunctional nonwovens: Robust noise reduction, abrasion resistance and antistatic electricity. Textile Research Journal, 2018, 88(22):2568-2578.

3. Kymakis E, Stratakis E, Stylianakis MM, Koudoumas E, Fotakis C. Spin coated graphene films as the transparent electrode in organic photovoltaic devices. Thin Solid Films, 2011, 520:1238-1241.

4. HongFei Shi, Can Wang, ZhiPei Sun,YueLiang Zhou, Kuijuan Jin, GuoZhen Yang. Transparent conductive reduced graphene oxide thin films produced by spray coating. Science China Physics, Mechanics \& Astronomy, 2015, 58(1):1-5

5. Zhang Y-L, Guo L, Xia H, Chen Q-D, Feng J, Sun H-B. Photoreduction of Graphene Oxides: Methods, Properties, and Applications. Advanced Optical Materials, 2013, 2(1):10-28.

6. McAllister $M J$ et al. Single Sheet Functionalized Graphene by Oxidation and Thermal Expansion of Graphite. Chem. Matter, 2007, 19:4396-4404.

7. Stankovich $\mathrm{S}$ et al. Synthesis of graphenebased nanosheets via chemical reduction of exfoliated graphite oxide. Carbon, 2007, 45(7):1558-1565.

8. Rabchinskii MK, Shnitov VV, Dideikin AT, Aleksenskii AE, Vul' SP, Baidakova MV, Pronin II, Kirilenko DA, Brunkov PN, Weise J, Molodtsov SL. Nanoscale Perforation of Graphene Oxide during
Photoreduction Process in the Argon Atmosphere. The Journal of Physical Chemistry C, 2016, 120(49):28261-28269.

9. Gengler RYN, Badali DS, Zhang D, Dimos K, Spyrou K, Gournis D, Miller RJD. Revealing the ultrafast process behind the photoreduction of graphene oxide. Nature Communications, 2013, 4(1).

10. Röthlisberger P, Gasse C, Hollenstein M. Nucleic Acid Aptamers: Emerging Applications in Medical Imaging, Nanotechnology, Neurosciences, and Drug Delivery. Int J Mol Sci., 2017, 18(11):E2430.

11. Park KS. Nucleic acid aptamer-based methods for diagnosis of infections. Biosens Bioelectron., 2018, 102:179-188.

12. Shigdar S et al. Aptamers as theranostic agents: modifications, serum stability and functionalization. Sensors (Base), 2013, 13(10):13624-13637.

13. Pu F, Ren J, QuX. Nucleobases, nucleosides, and nucleotides: versatile biomolecules for generating functional nanomaterials. Chem Soc Rev., 2018, 47(4):1285-1306.

14. Hughes RA, Ellington AD. Synthetic DNA Synthesis and Assembly: Putting the Synthetic in Synthetic Biology. Cold Spring Harb Perspect Biol., 2017, 9(1):023812.

15. Alexandrov GN, Smagulova SA, Kapitonov AN, Vasil'eva FD, Kurkina II, Vinokurov PV, Timofeev VB, Antonova IV. Thin partially reduced oxide-graphene films: Structural, optical, and electrical properties. Nanotechnologies in Russia, 2014, 9(7-8):363368.

16. Struchkov NS, Kondrashov VA, Rozanov RY, Nevolin VK. Research and development of the method of graphene oxide thin films local reduction by modulated laser irradiation. 18th Russian 
Youth Conference on Physics of Semiconductors and Nanostructures, Opto- and Nanoelectronics, 2016, Peter Great St Petersburg Polytechn Univ, St Petersburg, RUSSIA, 2016, vol. 816, BRISTOL: Iop Publishing Ltd, 2017. 17. Yamaguchi $H$, Eda G, Mattevi $C$ et al. Highly uniform $300 \mathrm{~mm}$ wafer-scale deposition of single and multilayered chemically derived graphene thin films. ACS Nano, 2010, 4:524-528.

18. Bock LC, Griffin LC, Latham JA, Vermaas EH, Toole JJ. Selection of singlestranded DNA molecules that bind and inhibit human thrombin. Nature, 1992, 355(6360):564-566.

19. Pica A, Krauss RI, Merlino A, Nagatoishi S, Sugimoto N, Sica F. Dissecting the contribution of thrombin exosite I in the recognition of thrombin binding aptamer. FEBS J., 2013, 280:6581-6588.

20. Musumeci D, Oliviero G, Roviello GN, Bucci EM, Piccialli G. G-quadruplexforming oligonucleotide conjugated to magnetic nanoparticles: synthesis, characterization, and enzymatic stability assays. Bioconjug Chem., 2012, 23(3):382-391.

21. Yang D. et al. Chemical analysis of graphene oxide flms afer heat and chemical treatments by X-ray photoelectron and microRaman spectroscopy. Carbon, 2009, 47:145-152.

22. Ferrari AC et al. Raman spectrum of graphene and graphene layers. Phys. Rev. Lett., 2007, 97:187401-187404.

23. Kotakoski J et al. From point defects in graphene to two-dimensional amorphous carbon. Phys. Rev. Lett., 2011, 106:105505105508.

24. Malard LM, Pimenta MA, Dresselhaus G, Dresselhaus MS. Raman spectroscopy in graphene. Phys. Rep., 2009, 473:51-88.

25. Kurapati R, Bonachera F, Russier J et al. Covalent chemical functionalization enhances the biodegradation of graphene oxide. 2D Mater., 2018, 5:015020.

26. Rodriguez-Silva AA, Movil-Cabrera O, Oliveira dos Anjos CT, Staser JA. Supercapacitor-Based Biosensor for Low Density Lipoprotein Detection. Journal of The Electrochemical Society, 2016, 163(6):B256-B263.

27. Lee D, Cho H, Han D, Chand R, Yoon T, Kim Y. J. Mater. Chem. B, 2017, DOI: 10.1039/C6TB03357A.

28. Bhavana Gupta, Niranjan Kumar, Kalpataru Panda et al. Role of oxygen functional groups in reduced graphene oxide for lubrication. Scientific Reports, 7:45030. DOI: 10.1038/srep45030.

29. Eluyemi MS, Eleruja MA, Adedeji AV et al. Synthesis and Characterization of Graphene Oxide and Reduced Graphene Oxide Thin Films Deposited by Spray Pyrolysis Method. Graphene, 2016, 5(03):143-154.

30. Akbar Bagri, Cecilia Mattevi, Muge Acik et al. Structural evolution during the reduction of chemically derived graphene oxide. Nature Chemistry, 2010, 2:581-587.

31.Xitong Liu, Chenxu Yan, and Kai Loon Chen. Adsorption of Human Serum Albumin on Graphene Oxide: Implications for Protein Corona Formation and Conformation. Environ. Sci. Technol., 2019, 53:8631-8639. 